\title{
More than ten years without any detectable HIV viremia: exceptionally long-term non-progressive HIV infection
}

\author{
Roberto Manfredi", Sergio Sabbatani, Giovanni Fasulo, Ciro Fulgaro \\ From $16^{\text {th }}$ International Symposium on HIV and Emerging Infectious Diseases \\ Marseille, France. 24-26 March 2010
}

\section{Background}

It is still unclear whether the virological-immunological steady-state observed among the small number of HIVinfected patients defined "long-term non-progressors" may have a prolonged-undefined duration, or it simply represents the extremity of a gaussian curve, and the progression of HIV disease is therefore an unavoidable event. These subjects are actively investigated, to understand this prolonged infection latency, so that many studies focused on the viremia levels predictive of disease progression.

\section{Methods}

A 42-year-old asymptomatic patient is followed since the age of 23 (year 1990), for her HIV infection.

\section{Results}

During periodic monitoring, carried out at least quarterly, HIV viremia always remained under the detectability threshold ( $<200$ copies/mL until 2003, $<40$ copies/mL since 2004), in absence of any viremia "blip", while the CD4+ T-lymphocyte count ranged from 597 and 794 cells $/ \mu \mathrm{L}$ (with a percentage of $28 \%$ to $36 \%$ of overall CD4+ $\mathrm{T}$-lymphocytes), concurrently with an always contained expansion of CD8+ T-lymphocyte subset (52\% to $56 \%$ during the entire follow-up). A genotypic resistance testing was never feasible, due to the persistingly undetectable viremia.

\section{Discussion}

In our patient followed since the age of 23 (year 1990), an HIV infection repeatedly confirmed by Western Blot assays was never associated with a detectable viremia, or a quantitative drop of CD4+ cell count, while the CD4+ percentage, although proportionally elevated (28-36\%), always remained below that of CD8+ lymphocytes, which had only a moderate expansion (52-56\%). Rodés et al. [AIDS 2004;18:1109] assessed five "long-term non-progressor" patients with a persistingly negative viremia during 6 years (1997-2003), also demonstrating a reduction of replication capacities related to the retrieval of R5 HIV strains, or the R77Q mutation of viral gene "vpr", while an homozygosis for the delta-32 variant of the CCRC co-receptor was not found. From the immunological point of view, the Author underlined a reduced expansion of CD8+ lymphocyte subset in these five subjects [AIDS 2004;18:1109].Case reports like ours, although very infrequent and therefore not representative of the entire population of HIV-infected individuals, should deserve in-depth virological and immunological assessment, on the ground of the present, enlarged investigation perspectives, to collect further informations on the network which sustains and allows a so prolonged clinical-immunological HIV infection latency.

Published: 11 May 2010

doi:10.1186/1742-4690-7-S1-P41

Cite this article as: Manfredi et al:: More than ten years without any detectable HIV viremia: exceptionally long-term non-progressive HIV infection. Retrovirology 2010 7(Suppl 1):P41.

* Correspondence: Roberto.manfredi@unibo.it

Infectious Diseases, S. Orsola Hospital, Bologna, Italy 